\section{Beate Kierey}

Die Behandlung und Versorgung von oberflächlichen Wunden ist ein Thema, das Menschen zu allen Zeiten beschäftigt hat. Verletzungen der Haut bei der Jagd, bei der täglichen Arbeit und bei kriegerischen Auseinandersetzungen verlangten von jeher nach einer heilenden Behandlung.

Ist es doch die Haut, die uns nach aussen abschirmt, eine Grenze zwischen «Äusserem und Innerem» darstellt und über die Fähigkeit verfügt, ihre Unversehrtheit selbstständig wieder herzustellen, indem eine natürliche Wundheilung einsetzt.

Diese «Heilkräfte, die jedem lebendigen Organismus innewohnen» [1], werden mit jeder speziellen Wundversorgung und -behandlung gefördert. Zum einen versucht man, im Wundbereich Bedingungen und ein Milieu herbeizuführen, das dem Wirken dieser Heilkräfte entgegenkommt, zum anderen versucht man, diese Heilkräfte durch entsprechende Anwendungen und Substanzen direkt anzuregen [1].

Herausfordernder wird die Wundbehandlung, wenn es sich um eine chronische Wunde handelt, also einen Defekt der Haut, der innerhalb von 8 Wochen unter einer üblichen Behandlung nicht heilt.

\section{Chronische Wunden}

Dies kann der Fall sein bei einer fortschreitenden Gewebszerstörung, die infolge venöser, arterieller oder stoffwechselbedingter Gefässleiden auftritt - wie beispielsweise schlecht heilende diabetische Wunden, Ulcera

\title{
Wundbehandlung mit Honig in der ambulanten Pflege
}

cruris oder Druckschädigungen wie etwa bei einem Dekubitus. Entsprechend dieser Krankheitsursachen sind vor allem ältere Menschen, die in der ambulanten Pflege betreut werden, von ihnen betroffen. Allerdings gehören auch exulzerierende KarzinomWunden zu den chronischen Wunden.

Am Beispiel des Dekubitus lässt sich dabei eine interessante Entwicklung feststellen: Ein Dekubitus wurde in den 1960er-Jahren - so in den damaligen Ausbildungen zur Krankenschwester gelehrt - trocken behandelt, also geeist und geföhnt. Heute zeigen Studien, «dass Wunden bei feuchter Behandlung doppelt so schnell heilen. Seitdem wurden neue Verbandmaterialien für die Praxis entwickelt und gefertigt (...)» [2].

\section{Wundversorgung mit Honig}

Von Hippokrates sind rund 300 Rezepturen überliefert, in denen Bienenhonig eine Rolle spielt (Abb. 1). «Honig ist für die Menschen am aller-
Abb. 1. Bienenstock mit Einflugloch.

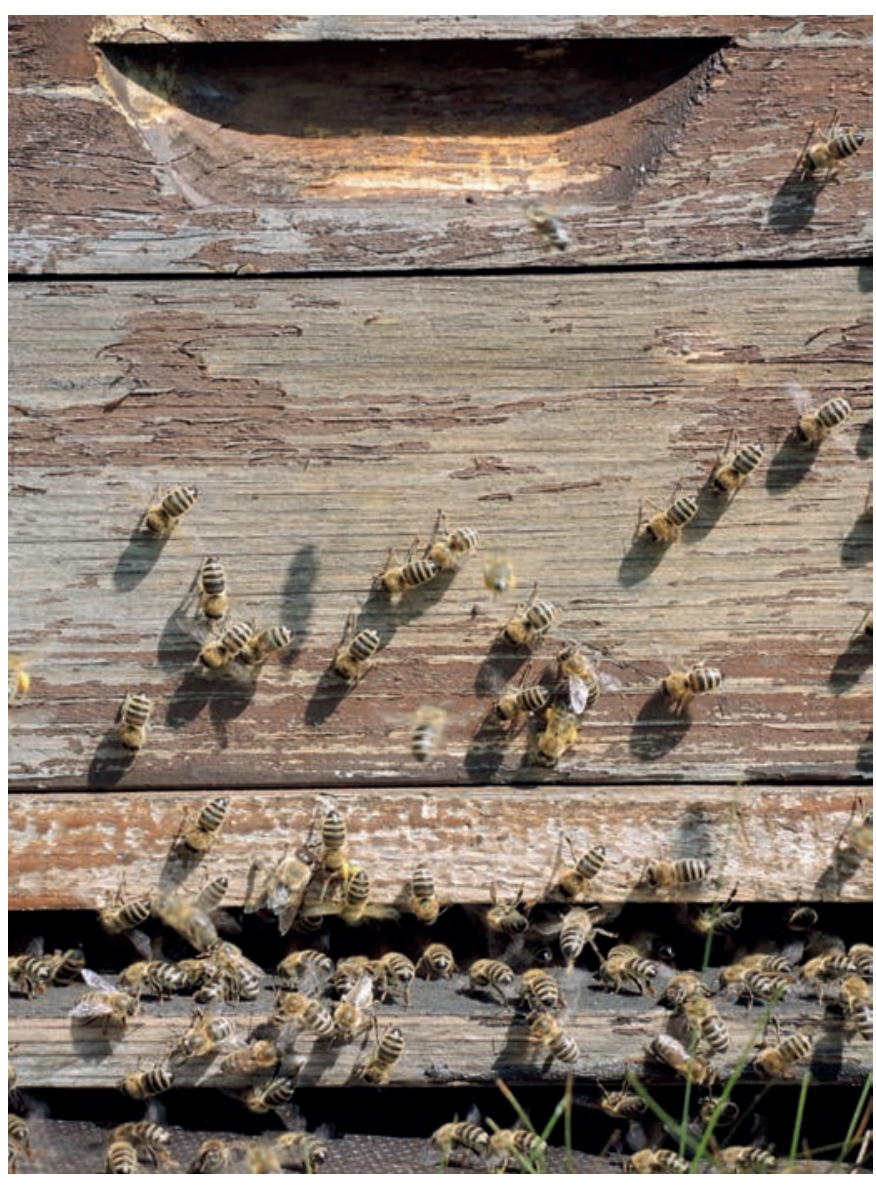

\section{KARGER}

๑๐ 2017 S. Karger GmbH, Freiburg

Fax +497614520714 
schönsten erschaffen, wenn er nur der Natur derselben entsprechend sowohl bei Gesunden wie bei Kranken zur richtigen Zeit und mit Mass angewendet wird» [3]. Wie in den Ausführungen zur «Geschichte der Wundbehandlung» [4] dargelegt, wird Honig seit dem Altertum durchgehend bis zur heutigen Zeit verwendet und mit pflanzlichen, tierischen oder mineralischen Stoffen kombiniert.

Bienen und andere Insekten haben in der Natur als Bestäuber eine ungleich wichtigere Rolle als diejenige, Honiglieferant für den Menschen zu sein. Honig ist ein natürlicher Zuckerstoff, der in vielen Zivilisationen schon über Jahrtausende Verwendung findet. Seine heilende Wirkung wird ebenfalls bereits in frühester Zeit beschrieben. Anwendung findet er in «unverändertem, rohen Zustand» bereits im Altertum, während er bei Hippokrates überwiegend «gekocht» verwendet wurde [4].

Im Mittelalter bis hin zur Neuzeit, schreiben Sänger et al. [4], wird der Honig sowohl in "gekochtem, verarbeiteten, wie im unverarbeiteten $\mathrm{Zu}$ stand verwendet», je nach Anwender und beabsichtigter Wirkung. Die sehr lange Anwendung mit Honig bei allen Arten von Wunden weist auf seine heilende Wirkung hin.

Vor allem die Reinigung, das Aufsaugen von ödemaler Flüssigkeit, die ausgleichende Wirkung auf das Immunsystem, die Förderung der Granulation, die Beschleunigung der Bildung von Zellgewebe, seine antimikrobielle Wirkung, die Geruchsminderung und Schmerzlinderung werden dabei immer wieder genannt [2].

Die antibakterielle Wirkung des Honigs ist zudem je nach gewählter Sorte sehr unterschiedlich; so wirkt Lindenhonig bei der Wundbehandlung intensiver als Akazien- oder Obstblütenhonig, und im Supermarkt erworbener Kleehonig zeige keine antibakterielle Wirkung [2].

\section{Unverträglichkeiten und Belastungen}

Seit einigen Jahren stösst die Verwendung von Honig bei der Wundbehandlung wieder auf grosses Interesse, denn Antibiotika-resistente Keime sind sowohl in der stationären als auch in der ambulanten Versorgung von Wunden zu einem grösseren und mit klassischen Mitteln nur schwer zu behandelnden Problem geworden.

Bei aller Wertschätzung des Honigs als Heilmittel ist jedoch auch grundsätzlich zu beachten, dass es bei der Anwendung zu einer Unverträglichkeit kommen kann. Leider kann Honig, je nach gewähltem Standort der Bienenvölker, inzwischen teilweise auch mit Pestiziden, Umweltgiften aller Art, Pollen oder Bakteriensporen belastet sein. So enthält er auch häufig neben nicht pathogenen Erregern Sporen von Clostridien. Zudem sollte bei der Anwendung in der Wundbehandlung Honig zum Einsatz kommen, der aus Bienenkörben stammt, «die nicht mit Arzneimitteln behandelt und möglichst in Gegenden aufgestellt worden sind, in denen keine Pestizide ausgebracht werden», führt Glaser [2] aus. Deshalb ist eine Nachfrage beim Imker unerlässlich bzw. sollte nur Honig mit Bio-Zertifikaten (Demeter) eingesetzt werden.

Der Markt bietet inzwischen auch sogenannten «medizinischen» Honig an (Gamma-bestrahlt wegen potenzieller anaerober Keime), um eventuelle Risiken für den Patienten zu minimieren. Beim medizinischen Honigprodukt muss nachgewiesen sein, dass es keine Kontamination enthält. $\mathrm{Zu}$ den medizinischen Honigen zählt unter anderem auch der Manuka-Honig, ein von Honigbienen aus dem Blütennektar der Südseemyrte ( $M a-$ nuka) erzeugter Honig, der in Neuseeland traditionell als Naturheilmittel verwendet wird.

In einem Informationsblatt des Universitätsklinikums Freiburg im Breisgau heisst es, dass für die Wundbehandlung «nur für den Zweck zuge- lassene sterile Medizinprodukte» [5] verwendet werden sollen. So können honighaltige Verbände Schmerzen durch Osmose oder den hohen Gehalt an Methylglyoxal verursachen, wie er z.B. im Manuka-Honig enthalten ist. Aufgrund einer möglichen Schmerzbelastung wird dem honighaltigen Wundbehandlungsmittel gar eine Negativempfehlung ausgesprochen [5]. Hersteller von honighaltigen Wundauflagen versuchen die Schmerzentstehung beispielsweise durch geringere Honigkonzentrationen oder dünne Honigbeschichtungen zu reduzieren.

«Eine antibiotische Wirkung von Honig (auch bei multiresistenten Keimen) wurde in vitro nachgewiesen", heisst es im Informationsblatt weiter; «im Tierversuch wurde eine antibiotische Wirkung sowie eine beschleunigte Abheilung von infizierten Wunden belegt». (In den bisherigen Wundheilungen in randomisierten kontrollierten Studien beim Menschen hat sich keine signifikant schnellere Wundheilung im Vergleich zu anderen Verbänden der feuchten Wundbehandlung gezeigt [5].)

Sänger et al. schliessen ihre Arbeit über die "Geschichte der Wundbehandlung» mit folgender Feststellung: «Viele der pharmakologischen Produkte benutzen zwar Honig als Grundstoff, bearbeiten ihn aber so stark, dass er nach der Honigverordnung nicht mehr als Honig bezeichnet werden kann.» Hieraus ergibt sich für sie die Notwendigkeit, «weitere Forschungen mit natürlichem, unverfälschten Honig» fortzuführen, «um (...) nachzuweisen, warum und wie Honig wirkt» [4].

\section{Aus der Praxis für die Praxis}

Auch in der ambulanten Pflege der Lukaspflege e.V. in Freiburg im Breisgau, die um die anthroposophische Pflege erweitert ist, wird in einigen Fällen bei der Wundversorgung Honig verwendet. Gemeinsam wurde die Handlungsleitlinie «Wundversorgung» erarbeitet. 
Die Pflegefachkräfte versuchen im Wundbereich Bedingungen zu schaffen und ein Milieu herbeizuführen, das dem Wirken der körpereigenen Heilkräfte entgegenkommt und diese durch entsprechende Anwendungen und Substanzen direkt anregt. Dazu berücksichtigen die Pflegefachkräfte, nach ärztlicher Anordnung, zusätzlich allgemeine Massnahmen zur Förderung der Wundheilung - wie unter anderem die Lagerung und Mobilisation des Patienten, das Wickeln der Beine, das Anziehen von Kompressionsstrümpfen, aber auch die Förderung der Compliance; z.B. durch eine Änderung der Ernährungsgewohnheiten und des Körpergewichts usw.

Die Wunde und der Heilungsverlauf werden in der Wunddokumentation beschrieben. Nach der «Ersterhebung» wird alle 7 Tage oder bei gravierenden Veränderungen sofort dokumentiert. Mit Einverständnis des Patienten wird zeitgleich eine Fotodokumentation (gleiche Perspektive und Wundlineal) vorgenommen und dies entsprechend archiviert.

\section{Praxisbeispiel: Dekubitus- versorgung mit Honig}

Ein 55-jähriger Patient wird - nach massiver Hirnblutung im Januar 2014 nach seinem Krankenhausaufenthalt und nachgehender Rehabilitation mit einem positiven MRSA(Methicillinresistenter Staphylococcus aureus)-Befund - Mitte September 2014 nach Hause entlassen. Hier wird er durch seine Ehefrau und den ambulanten Pflegedienst der Lukaspflege e.V. betreut. $\mathrm{Zu}$ diesem Zeitpunkt bestehen eine Hemiparese rechts, eine Aphasie und ein stark gestörter Wärmehaushalt. Die rechte Schulter ist subluxiert und instabil. Neben einer vollständigen Harn- und Stuhlinkontinenz neigt der Patient zu Mykosen im Intimbereich.

Bei der Übernahme in die häusliche Pflege zeigt sich am Steiss ein weisslicher, zerfranster Dekubitus von ca. 1,2 $\mathrm{cm}$ Durchmesser und $2 \mathrm{~mm}$ Tiefe mit granulierendem Wundgrund und feuchten Wundrändern. Die Wunde ist von aufgeweichter Haut umgeben. Täglich wird die Wunde gereinigt (Tender Wet ${ }^{\circledR}$ ) und zuerst mit Allevyn life $^{\circledR}(7,5 \times 7,5)$ versorgt. Die Wundumgebung wurde begleitend mit WELEDA Rosmarinsalbe 10\% rhythmisch eingerieben, um die Durchblutung und das Durchwärmen der umgebenden Haut anzuregen.

Die Besiedlung mit MRSA wird unter anderem auch im gesamten Intimbereich und Dekubitus nachgewiesen. Anfänglich ist eine grosse Erschöpfung des Patienten festzustellen. Nach Transfer in den Rollstuhl sitzt er täglich bis zu maximal $3 \mathrm{~h}$ im Rollstuhl. Starkes Schwitzen, eine erhöhte Urinausscheidung und Druck auf die Sitzbeinhöcker durch das Sitzen erschweren die Wundheilung und vergrössern den Dekubitus anfänglich.

Regelmässige Ruhephasen im Bett und konsequente Lagerung zur Entlastung folgen. Nach Absprache und mit Einverständnis des behandelnden Hausarztes erfolgt eine konsequente MRSA-Sanierung mit Thymianöl (Nasenöl) und -tee (innerlich und äusserlich). Die Wundversorgung des Dekubitus wird zudem mit Alginatkompressen und Honig vorgenommen.

Alginatkompressen bestehen aus tamponierbaren, wirkstofffreien Cal-
cium-Alginat-Fasern, die sich im Austausch mit Natriumsalzen von Blut oder Sekret in ein hydrophiles, strukturstabiles, nicht mit der Wunde verklebendes Gel umwandeln. Wegen seines intrakapillären Saugvermögens werden überschüssiges Wundexsudat und Keime aufgenommen und so durch den regelmässig erfolgten Verbandswechsel entfernt [6].

Der Honig wurde von der Pflegefachkraft nun direkt auf die Alginatkompresse aufgetragen, dann direkt auf den Dekubitus aufgebracht und in regelmässigen Abständen erneuert.

Im Verlauf nehmen die Wundtiefe und die Menge des Exsudats deutlich ab; ebenfalls klingt die Rötung der Wundumgebung sichtbar ab. Mitte Dezember 2014 ist der Dekubitus am Steiss geschlossen, und es erfolgt anschliessend eine Pflege der Haut mit Panthenolsalbe plus Zink und einer konsequenten Dekubitusprophylaxe.

Insgesamt konnte das Aufsaugen von ödemaler Flüssigkeit, die Förderung der Granulation, die Beschleunigung der Bildung von Zellgewebe und eine ausgleichende Wirkung auf das Immunsystem, auch durch die Thymian-Therapie, bei der Behandlung mit Alginat und Honig festgestellt werden.

\section{Fazit}

Es bleibt für die Praxis und den weiteren Einsatz von Honig in der Wundversorgung $\mathrm{zu}$ wünschen, dass $\mathrm{zu}$ künftig verstärkt Forschungen zur Wirkungsweise und Anwendung von Honig erfolgen, so wie dies auch von Sänger et al. [4] in ihrer Veröffentlichung angeregt wurde.

\section{Literatur}

1 Goyert A: Vorwort; in Glaser H: Erfolgreiche Wundbehandlung. Aus der Praxis der anthroposophisch erweiterten Krankenpflege. Stuttgart, Urachhaus, 2000, p 9.

2 Glaser H: Erfolgreiche Wundbehandlung. Aus der Praxis der anthroposophisch erweiterten Krankenpflege. Stuttgart, Urachhaus, 2000.

3 Beck T: Hippokrates Erkenntnisse, Im griechischen Text ausgewählt und übersetzt. Jena, Eugen Diederichs, 1907.

4 Sänger A, Fretz A, David E: Geschichte der Wundbehandlung mit Honig. Z Allgemeinmedizin 2015;91:187-192.

\footnotetext{
5 Informationsblatt 9. Freiburger Wundsymposium, 12.10.2016, Bad Krozingen.

6 von Hallern B: Kompendium Wundbehandlung. Wirkungsweise und Indikation von Wundbehandlungsprodukten. Stade, 2006.
} 